\section{Dorin Ritzmann}

\section{Einleitung}

Welche Behandlungsmöglichkeiten bietet die Phytotherapie bei Endometriose-Erkrankungen? Um auf diese Frage einzugehen, müssen die Eigenheiten der Endometriose, aber auch die Komplexität der individuellen Rezeptur erläutert werden.

Beginnen möchte ich mit einem typischen Fallbeispiel. Frau C., 33 Jahre alt, meldet sich per E-Mail in meiner gynäkologisch-geburtshilflichen Facharztpraxis. Sie wünscht eine phytotherapeutische Behandlung ihrer Endometriose. Sie hat bereits eine lange Leidensgeschichte hinter sich: Als Jugendliche litt sie stark unter Dysmenorrhö und nahm schon früh kombinierte Ovulationshemmer ein. Mit 30 Jahren stoppte sie diese aus Kinderwunschgründen. Die Schmerzen vor und während den Menstruationsblutungen wie auch die Blutungsstärke und -dauer nahmen deutlich zu. Es traten Harnwegsinfektionen, Zwischenblutungen und lange Vorblutungen sowie Schmerzen bei der Sexualität auf. Mehrere Konsultationen beim Hausarzt und auf medizinischen Notfallstationen folgten, ebenso gynäkologische Abklärungen. Es wurde ein seelisch mitbedingtes Schmerzsyndrom diagnostiziert; starke Schmerzmittel wurden empfohlen.

Anlässlich eines Betroffenenberichtes in einer Illustrierten wurde Frau C. auf das Krankheitsbild Endometriose aufmerksam. Sie liess sich von ihrem Hausarzt an einen spezialisierten Gy-

\title{
Phytotherapeutische Behandlungsmöglichkeiten bei Endometriose-Erkrankungen
}

näkologen überweisen. Dieser vermutete aufgrund der Beschwerden eine schwere Endometriose, die operativ bestätigt wurde. Die jahrelange Leidenszeit der Betroffenen bis zur korrekten Diagnose ist leider bis heute typisch bei Endometriose. Bei Frau C. handelte es sich um eine peritoneale Endometriose mit Ummauerung eines Ureters und konsekutiver Hydronephrose, obliteriertem Douglas-Raum, Endometriomata beider Ovarien und motilitätsveränderten Tuben beidseits. Die Operation verlief günstig mit Befreiung des Ureters. Frau C. wie auch ihr Ehemann lehnten die empfohlene Hysterektomie mit Adnexektomie als Lösung des Problems ab, da sie Kinder wünschten.

Es wurde eine Behandlung mit Gonadotropin-Releasing-Hormonen (GnRH) (Zoladex) und Add-BackÖstrogentherapie (Estrafem) sowie Aromatasehemmern (Arimidex) durchgeführt; danach fanden sieben In-vitro-Fertilisationszyklen ohne positives Ergebnis statt. Nach 2,5 Jahren brach das Ehepaar diesen körperlich, seelisch und finanziell belastenden Weg ab. Postoperativ verbesserte sich die Endometriose-Symptomatik in Bezug auf Harnwegsinfektionen und Schmerzen bei Sexualität deutlich; die starken Blutungen wie auch die Dysmenorrhö blieben allerdings bestehen. Frau C. schaute sich nach komplementärmedizinischen Verfahren um und begann eine Shiatsu-Therapie neben meditativen Techniken. In dieser Situation meldete sich Frau C. bei mir.

\section{Was ist Endometriose?}

Endometriose ist eine häufige Erkrankung bei Frauen zwischen Menarche und Menopause mit einer Prävalenz von $6 \%[1,2]$ bis $15 \%[3,4]$. Etwa die Hälfte der Betroffenen, also etwa 5\% der weiblichen Bevölkerung Europas und Nordamerikas, leidet an Endometriose-assoziierten Beschwerden. Diese treten oft bereits zum Zeitpunkt der ersten Menstruation auf und nehmen mit den Jahren stetig zu. Mit der Menopause sistiert die Endometriose-Aktivität. Folgeprobleme wie Vernarbungen, Adhäsionen und Kinderlosigkeit bleiben jedoch bestehen.

\section{Beschwerden}

Die chirurgisch-anatomische Einteilung der Endometriose (Endometriosis Fertility Index (EFI)) $[5,6]$ ist aus phytotherapeutischer Sicht unpraktisch. In Bezug auf eine erfolgversprechende Behandlung können drei Endometriose-Beschwerdegruppen unterschieden werden [7]:

- Die Adenomyose (Endometriosis genitalis interna) (Abb. 1) führt oft zu starken dysmenorrhoischen Beschwerden und Schmierblutungen. Sie zeigt sich sonografisch als kugelige Gebärmutter mit subendometrialen Gefässzeichnungen.

- Die ovarielle Endometriose (Endometriosis genitalis externa) (Abb. 2) führt eher zu Druckbeschwerden im Unterbauch und verläuft oft be-

\section{KARGER}

(C) 2017 S. Karger GmbH, Freiburg 


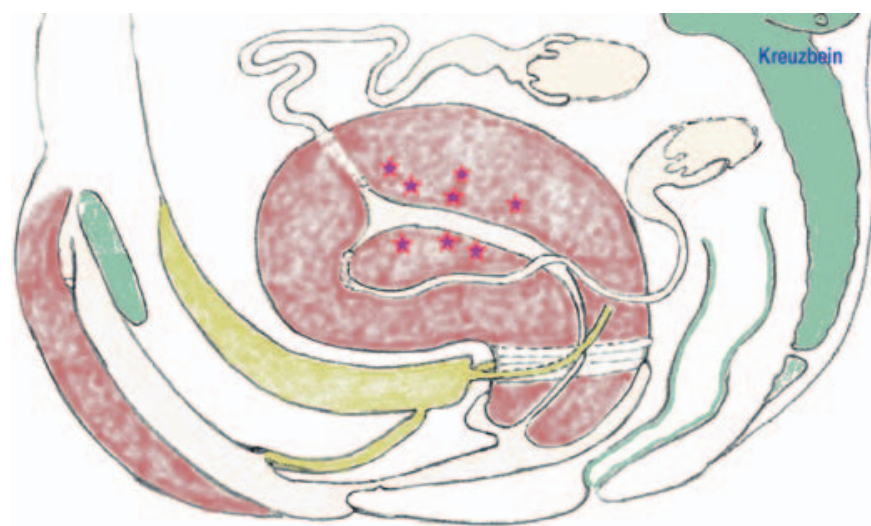

Abb. 1. Adenomyose

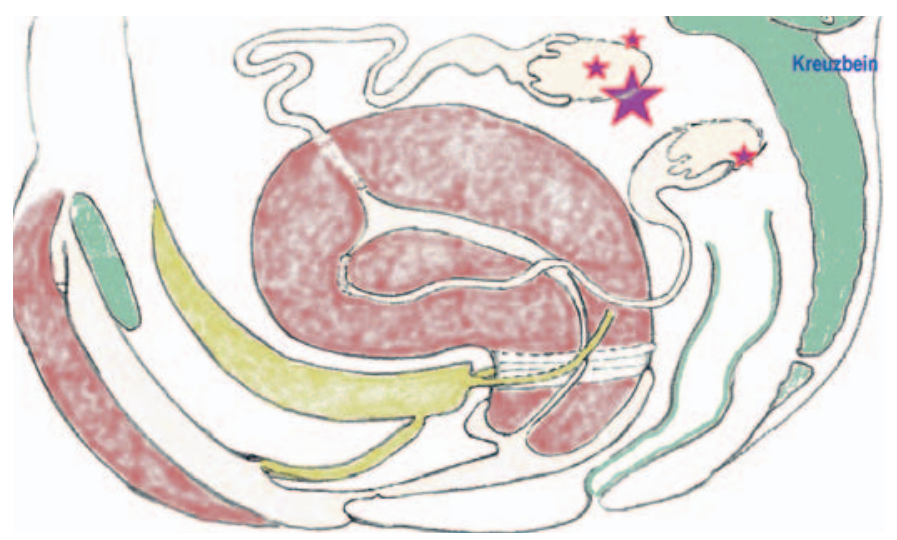

Abb. 2. Ovarielle Endometriose. schwerdearm. Sonografisch zeigen sich intraovarielle Endometriomata unterschiedlicher Grösse.

- Die peritoneale Endometriose (Endometriosis genitalis externa und Endometriosis extragenitalis) (Abb. 3) führt perimenstruell $\mathrm{zu}$ hochakuten Schmerzattacken, vergleichbar mit den Schmerzen einer Appendizitis. Sie kann zu einer Mesothel-Veränderung führen, die $\mathrm{Fi}$ broblast-ähnlich tiefere Gewebe und Organe infiltriert und vernarbt [8]. Sie ist meist weder sonografisch noch mittels Magnetresonanztomographie darstellbar, was zu erheblichen Verzögerungen in der korrekten Diagnosestellung führen kann.

Da es sich bei der Endometriose um eine funktionelle Störung junger Frauen handelt, sind diagnostische Schritte gut zu überlegen. Einer korrekten operativen Diagnose stehen Risiken der operativen Verfahren und fehlende nachhaltige Therapieoptionen gegenüber [9]. Die Einordnung der Beschwerden in eine bekannte Erkrankung bringt den Betroffenen meist eine grosse Erleichterung. Die Festlegung der Graduierung auf der Basis chirurgisch-anatomischer Befunde mit ihren deprimierenden Prognosen in Bezug auf Schmerzverminderung und Fruchtbarkeit kann jedoch zur Erschwerung der Selbstregulation und Selbstheilung führen.

\section{Konventionelle Therapien}

Nachweisbar wirksame Methoden zur Schmerzlinderung bei Endometriose sind laut Cochrane Database [9] aus dem Jahr 2014 die Behandlung mit GnRH-Analoga, die wegen erheblicher Nebenwirkungen als langfristig ungünstig beurteilt werden, das in der Schweiz wegen seinen Nebenwirkungen nicht mehr zugelassene, anabole Danazol und die Einlage eines Levonorgestrel-Intrauterinsystems. Chirurgische Exzisionen von Endometriomata sind wirksam und für die ersten 9-12 Monate ablativen Verfahren überlegen. In Bezug auf Fruchtbarkeit sind medikamentöse Therapien - mit Ausnahme einer dreimonatigen GnRH-Behandlung vor einer künstlichen Befruchtung - ohne belegbaren Nutzen. Mifepriston-Behandlungen wurden hier wegen fehlender Kontrollstudien nicht einbezogen; dies gilt auch für neue Therapieansätze auf immunologischem Niveau, die erst in Erforschung sind [10].

\section{Alternative und komplementäre Therapieoptionen}

In Übereinstimmung mit aktuellen Forschungsergebnissen wird die Endometriose in der traditionellen $\mathrm{Me}-$ dizin als entzündlich-proliferative Ganzkörpererkrankung betrachtet. Hieraus ergeben sich einige grundlegende Behandlungsstrategien:
- Eine Reduktion von Entzündungsförderern wird generell empfohlen, insbesondere ein Verzicht auf Nikotin und Zucker in jeder Form.

- Eine Sättigung mit Entzündungshemmern wird angestrebt durch vermehrte Aufnahme von Lignanen (Leinöl) und aktiven Polyphenolen in frischen Blättern (Green Medical Smoothie / Grünsaft).

- Eine Re-Integration der schmerzenden Körperregion «Becken» kann durch manuelle Techniken bei Beckenringdysfunktionen sowie durch Tanz, Yoga (Luna-Yoga), Hypno-Analgesie und Körpertherapien erreicht werden.

Die Phytotherapie geht von den spezifischen Bedürfnissen der Betroffenen aus. Drei Hauptpflanzen werden je nach Beschwerdegruppe ausgesucht und mit Nebenpflanzen kombiniert, die individuell zugefügt werden.

Zur Behandlung einer reinen Adenomyose verwende ich die Hauptpflanzen Hamamelis virginiana Folium et Cortex (Virginische Zaubernuss), Achillea millefolium Herba (Gemeine Scharfgarbe) und Plantago lanceolata Herba (Spitzwegerich). Bei Betroffenen von ovarieller Endometriose genügen meist entzündungshemmende Massnahmen. Die peritoneale Form der Endometriose muss oft operativ und medikamentös vorbehandelt werden, um allfällige Vernarbungen zu entfernen und das Gesamtvolumen an Endometriose-Her- 


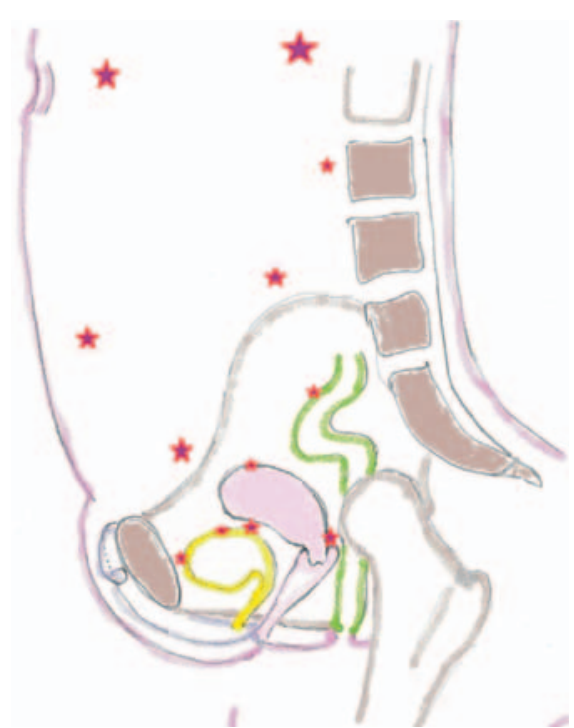

Abb. 3. Peritoneale Endometriose.

den zu vermindern. Danach kann die Phytotherapie zur nachhaltigen Beschwerdelinderung beitragen. Als Hauptpflanzen verwende ich Sabal serrulata Fructus (Sägepalme), Verbascum densiflorum Flos (Grossblütige Königskerze) und Plantago lanceolata Herba (Spitzwegerich).

\section{Ätiologische Erkenntnisse}

Die Endometriose wird als eine proliferativ-entzündliche Erkrankung angesehen [2, 11]. Ätiologisch wird eine biochemische Prägung während der Schwangerschaft und perinatal diskutiert $[12,13]$. Eine Belastung mit Xeno-Östrogenen ist mit Endometriose und Myomata korreliert [10, 14]. Mit Beginn der Pubertät wird eine Entzündungskaskade ausgelöst, die über die Estradiol-Produktion gesteuert ist [2, 15-18]. Die biochemische Dysbalance führt oft zu Funktionsstörungen wie chronischen Schmerzen sowie Tubentransport- und Einnistungsproblemen. Anatomische Strukturveränderungen können, müssen je- doch nicht eine Folge sein $[8,19]$. Mit meinen Erfahrungen übereinstimmend, können schon minimale Änderungen in der Exposition zu deutlichen Veränderungen in der Endometriose-Aktivität führen [13].

\section{Konkrete Phytotherapie im Fallbeispiel}

Zurück zum Fallbeispiel: Frau C. hat zwei Anliegen - einerseits benötigt sie eine Reduktion der starken und schmerzhaften Menstruationen, andererseits wünscht sich das Ehepaar Kinder. Neben den erwähnten allgemeinen Massnahmen zur Entzündungsreduktion, die ich bei Kinderwunsch beiden Wunsch-Eltern empfehle, erhält Frau C. eine Urtinkturmischung mit Bryophyllum calanchoë Folium (Brutblätter), Daucus carota Flos et Fructus (Möhre), Betula alba Folium (Hänge-Birke) und Sabal serrulata Fructus (Sägepalme). Der Kinderwunsch-Vater erhält Bryophyllum calanchoë Folium (Brutblätter), Boswellia serrata Olibanum (Indischer Weihrauch) und Rubus idaeus Folium (Himbeere). Innerhalb von 3 Monaten kommt es zu einer intrauterinen Zwillingsschwangerschaft. Während der Schwangerschaft wird auf eine einfache Gebärmutter beruhigende $\mathrm{Mi}$ schung umgestellt (Bryophyllum calanchoë Folium, Betula alba Folium). Beide Kinder kommen reif und gesund zur Welt.

Nach der ersten Schwangerschaft und Stillzeit sind die Monatsblutungen etwas weniger schmerzhaft, verursachen jedoch noch immer Unterbauch- und Kopfschmerzen. Die phytotherapeutische Behandlung wird mit Hamamelis virginiana Folium et Cortex (Virginische Zaubernuss), Achillea millefolium Herba (Gemeine Scharfgarbe), Betula alba Folium
(Hänge-Birke), Vitex agnus-castus Fructus (Mönchspfeffer) und Sabal serrulata Fructus (Sägepalme) durchgeführt. Nach 4 Monaten sind keine Analgetika mehr notwendig. Einige Jahre später wünscht sich das Ehepaar noch ein Kind. Mit einer leicht angepassten Rezeptur ohne Sabal serrulata Fructus wird Frau C. innerhalb kurzer Zeit schwanger und kann ein reifes, gesundes Kind gebären. Nach dem dritten Kind stellt sich die Frage nach einer nachhaltigen Langzeitbehandlung der Endometriose. Die schmerzund blutungslindernde Urtinktur-Mischung wird mit Erigeron canadense Herba (Kanadisches Berufkraut) ergänzt. Damit kann Frau C. ein normales Leben führen.

\section{Fazit}

Endometriose ist eine dysfunktionelle Erkrankung, die schon auf kleine, gezielte Veränderungen im biochemischen Gleichgewicht mit deutlichen Verbesserungen reagieren kann. Es lohnt sich speziell bei diesem chronischen schweren Krankheitsbild, die phytotherapeutischen Möglichkeiten auszuschöpfen.

Für Interessierte gibt es mehrere Möglichkeiten, sich in gynäkologischer Phytotherapie weiterzubilden. Die Schweizerische Medizinische Gesellschaft für Phytotherapie (SMGP; www.smgp.ch) bietet ein Curriculum in der gesamten Phytotherapie an; die Untergruppe Herbadonna (www.herbadonna.ch) dient dem Austausch und der Anregung zur praktischen Umsetzung im Bereich Gynäkologie und Geburtshilfe. Ein vertieftes praktisches Erlernen einzelner gynäkologisch-geburtshilflicher Gebiete wird von meiner Praxis ab 2017 angeboten (www.phytogyn.ch). 


\section{Literatur}

1 Denny E, Mann CH: Endometriosis-associated dyspareunia: the impact on women's lives. J Fam Plann Reprod Health Care 2007;33: 189-193.

2 Miller JE, et al: Implications of immune dysfunction on endometriosis associated infertility. Oncotarget 2016;DOI: 10.18632/oncotarget.12577.

3 Culley L, et al: The social and psychological impact of endometriosis on women's lives: a critical narrative review. Hum Reprod Update 2013;19:625-639.

4 Dunselman GA, et al: ESHRE guideline: management of women with endometriosis. Hum Reprod 2014;29:400-412.

5 Adamson GD, Pasta DJ: Endometriosis fertility index: the new, validated endometriosis staging system. Fertil Steril 2010;94:1609-1615.

6 Tomassetti C, et al: External validation of the endometriosis fertility index (EFI) staging system for predicting non-ART pregnancy after endometriosis surgery. Hum Reprod 2013;28: 1280-1288.

7 Young VJ, et al: The role of the peritoneum in the pathogenesis of endometriosis. Hum Reprod Update 2013;19:558-569.
8 Ono YJ, et al: Estradiol-mediated hepatocyte growth factor is involved in the implantation of endometriotic cells via the mesothelial-tomesenchymal transition in the peritoneum. Am J Physiol Endocrinol Metab 2015;308: E950-E959.

9 Brown J, Farguhar C: Endometriosis: an overview of Cochrane Reviews. Cochrane Database Syst Rev 2014;3:CD009590.

10 Hart DA, et al: Curbing inflammation in multiple sclerosis and endometriosis: should mast cells be targeted? Int J Inflamm 2015;2015: 452095.

11 Harris HR, et al: Endometriosis and the risks of systemic lupus erythematosus and rheumatoid arthritis in the Nurses' Health Study II. Ann Rheum Dis 2016;75:1279-1284.

12 Crain DA, et al: Female reproductive disorders: the roles of endocrine disrupting compounds and developmental timing. Fertil Steril 2008;90:911-940.

13 Guo SW: Epigenetics of endometriosis. Mol Hum Reprod 2009;15:587-607.

14 Hunt PA, et al: Female reproductive disorders, diseases, and costs of exposure to endocrine disrupting chemicals in the European Union. J Clin Endocrinol Metab 2016;101:1562-1570.
15 Sofo V, et al: Correlation between dioxin and endometriosis: an epigenetic route to unravel the pathogenesis of the disease. Arch Gynecol Obstet 2015;292:973-986.

16 Attia GR, et al: Progesterone receptor isoform A but not B is expressed in endometriosis. J Clin Endocrinol Metab 2000;85:2897-2902.

17 González-Fernández R, et al: Expression levels of the oxidative stress response gene ALDH3A2 in granulosa-lutein cells are related to female age and infertility diagnosis. Reprod Sci 2016; 23:604-609.

18 Khan KN, et al: Estrogen and progesterone receptor expression in macrophages and regulation of hepatocyte growth factor by ovarian steroids in women with endometriosis. Hum Reprod 2005;20:2004-2013.

19 Monument MJ, et al: Neuroinflammatory mechanisms of connective tissue fibrosis: targeting neurogenic and mast cell contributions. Adv Wound Care (New Rochelle) 2015;4:137-151. 